\title{
Compressed PARAFAC Model-based Two-Dimensional Angle Estimation for Acoustic Vector-Sensor Arrays
}

\author{
Le $\mathrm{Xu}^{1, \mathrm{a}}$, You Sun ${ }^{1, \mathrm{~b}}, \mathrm{Na} \mathrm{Shi}^{1, \mathrm{c}}$, Xiaofei Zhang ${ }^{1,2,3, \mathrm{~d}}$
}

${ }^{1}$ College of Electronic and Information Engineering, Nanjing University of Aeronautics and Astronautics, Nanjing, 210016, People's Republic of China.

${ }^{2}$ State Key Laboratory of Millimeter Waves, Southeast University, Nanjing, China, 210096.

${ }^{3}$ National Mobile Communications Research Laboratory, Southeast University, Nanjing, China, 210096.

axule@nuaa.edu.cn, ${ }^{b}$ sunyou_nuaa@163.com,,'shina_nuaa@163.com, ${ }^{\text {d}} z$ hangxiaofei@nuaa.edu.cn

Keywords: arbitrary array, acoustic vector-sensor, compress, PARAFAC model, angle estimation.

\begin{abstract}
In this paper, in order to estimate the angles for arbitrarily spaced arrays with acoustic vector-sensor, we combine the compressed sensing theory with parallel factor (PARAFAC) model, and propose a neoteric angle estimation algorithm. The proposed algorithm firstly compressed the PARAFAC model, then exploit trilinear alternating least square (TALS) algorithm to estimate the parameter matrices and obtains the angle estimation. Owing to compression, the proposed algorithm has smaller storage requirement and lower computational complexity, compared with the conventional PARAFAC algorithm. It's also works well to achieve automatically paired azimuth and elevation angles. The angle estimation performance of the proposed algorithm is close to the conventional PARAFAC algorithm, and is better than the estimation of signal parameters via rotational invariance techniques (ESPRIT) algorithm. Various simulation results demonstrate the effectiveness of our algorithm.
\end{abstract}

\section{Introduction}

Compared with traditional sensor arrays, acoustic vector sensors can measure the acoustic pressure as well as all three orthogonal components of the acoustic particle velocity, which made it have greater advantages in collecting more acoustic information and enhancing the system performance. Ref.[1] have elaborated the foundation data model of an acoustic vector sensor array and now researchers on acoustic vector sensors mainly turned to the study of incoming signal direction of arrival (DOA) estimation, for which many DOA estimation algorithms have been proposed, including the Capon technique [2], estimation of signal parameters via rotational invariance technique (ESPRIT) [3], root multiple signal classification (MUSIC) [4], self-initiating MUSIC [5], propagator method (PM) [6] and so on. Parallel factor (PARAFAC), also called trilinear decomposition [7], first introduced as a data analysis tool in chemometrics and psychometrics [8], has been extended to signal processing and communication. In [9], PARAFAC method has been used to estimate the angle for acoustic vector-sensor Arrays, However, the conventional PARAFAC-based algorithm suffers from a heavy computational load.

Compressive sensing (CS) [10] has aroused considerable concern recently, and it has been successfully applied to image processing, radar imaging, channel estimation and some of other fields. An approach of multi-way compressed sensing for sparse and low-rank tensors was proposed in [10]. This approach, which combines the PARAFAC model with CS theory, reduces the computational complexity and saves memory capacity when compared with the conventional PARAFAC method.

Ref. [9] exploited the PARAFAC model to estimate the angle for acoustic vector-sensor arrays, and the PARAFAC model-based algorithm has good parameter estimation performance. However, it has high computational complexity and storage demand. In this paper, we improved the algorithm in [9] and joined the PARAFAC model compression process, which effectively reduced the algorithm complexity and storage requirement with very close angle estimation performance. 
Notation: $(.)^{*},(.)^{T}(.)^{H},(.)^{-1}$, and $(.)^{+}$stand for the operations of complex conjugation, transpose, conjugate-transpose, inverse, and pseudo-inverse, respectively. \|\|$_{F}$ and $\|_{0}$ denote Forbenius norm and $l_{0}-$ norm; $\otimes$ and $\circ$ are Kronecker product and Khatri-Rao product, respectively; $\operatorname{diag}(\boldsymbol{b})$ represents a diagonal matrix composed of a vector $\boldsymbol{b} ; D_{n}(\boldsymbol{A})$ stands for a diagonal matrix constructed out of the $n$th row of $\boldsymbol{A}$. min(.) is the operation to get the minimum element of an array.

\section{Data Model}

Assume that there are $K$ narrowband plane waves impinges on an array consisting of $M$ acoustic vector sensors, which all located at arbitrary unknown three dimensional positions as shown in Fig.1.

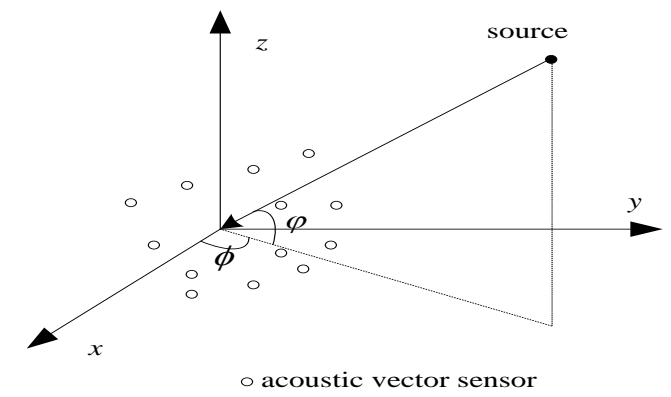

Figure 1. The structure of acoustic-vector sensor array

We assume the signals are all in the far-field and the noise is additive white Gaussian, which is independent of the sources. The arrival direction of the $k$ th signal is $\left(\phi_{k}, \varphi_{k}\right)$, where $\phi_{k}$ and $\varphi_{k}$ stand for the azimuth angle and the elevation angle, respectively. Let's denote the 2D-DOA of the $k$ th source as $\theta_{k}=\left[\phi_{k}, \varphi_{k}\right]^{T}$. According to [1], the output at the acoustic vector sensors array can be expressed as:

$$
\mathbf{x}(t)=[\mathbf{A} \circ \mathbf{H}] \mathbf{b}(t)+\mathbf{n}(t) .
$$

where $\mathbf{A}=\left[\mathbf{a}\left(\boldsymbol{\theta}_{1}\right), \mathbf{a}\left(\boldsymbol{\theta}_{2}\right), \ldots, \mathbf{a}\left(\boldsymbol{\theta}_{K}\right)\right] \in \mathbb{C}^{M \times K}$ is an $M \times K$ matrix composed of $K$ receive steering vectors. $\mathbf{b}(t)=\left[b_{1}(t), b_{2}(t), \cdots, b_{K}(t)\right]^{T} \in \mathbf{C}^{K \times 1}$ include $K$ sources. $\mathbf{n}(t)$ is the received additive white Gaussian noise vector. $\mathbf{H}=\left[\boldsymbol{h}\left(\boldsymbol{\theta}_{1}\right), \boldsymbol{h}\left(\boldsymbol{\theta}_{2}\right), \ldots, \boldsymbol{h}\left(\boldsymbol{\theta}_{k}\right)\right] \in \mathbb{C}^{4 \times K}$ in which:

$$
\boldsymbol{h}\left(\boldsymbol{\theta}_{k}\right)=\left[1, \cos \phi_{k} \cos \varphi_{k}, \sin \phi_{k} \cos \varphi_{k}, \sin \varphi_{k}\right]^{T}
$$

Then the received data matrix $\mathbf{X} \in \boldsymbol{C}^{4 M \times J}$ for $J$ snapshots is given by:

$$
\mathbf{X}=\left[\mathbf{x}\left(t_{1}\right), \mathbf{x}\left(t_{2}\right), \cdots, \mathbf{x}\left(t_{J}\right)\right]=[\mathbf{A} \circ \mathbf{H}] \mathbf{B}^{T}+\mathbf{N}_{x}=\left[\begin{array}{c}
\mathbf{X}_{1} \\
\mathbf{X}_{1} \\
\vdots \\
\mathbf{X}_{M}
\end{array}\right]=\left[\begin{array}{c}
\mathbf{H} D_{1}(\mathbf{A}) \\
\mathbf{H} D_{2}(\mathbf{A}) \\
\vdots \\
\mathbf{H} D_{M}(\mathbf{A})
\end{array}\right] \mathbf{B}^{T}+\mathbf{N}_{x} .
$$

where $\mathbf{B}^{T}=[\mathbf{b}(1), \mathbf{b}(2), \cdots, \mathbf{b}(J)] \in \mathbf{C}^{K \times J}$ is the signal source matrix, $\mathbf{N}=[\mathbf{n}(1), \mathbf{n}(2) \cdots, \mathbf{n}(J)] \in \mathbf{C}^{4 M \times J}$ is the noise matrix, and $D_{n}$ (A) denotes a diagonal matrix constructed out of the $n$th row of $\mathbf{A}$. Thus, $\mathbf{X}_{m}$ in (3) can be written as

$$
\mathbf{X}_{m}=\mathbf{H} D_{m}(\mathbf{A}) \mathbf{B}^{T}+\mathbf{N}_{m} \quad(m=1,2, \cdots, M)
$$

where $\mathbf{N}_{m}$ is the slice of the noise matrix. (4) is also denoted as the quadrilinear model 


$$
x_{m, p, j}=\sum_{k=1}^{K} a_{m, k} h_{p, k} b_{j, k}+n_{m, p, j}(m=1,2, \cdots, M ; p=1,2,3,4 ; j=1,2, \cdots, J)
$$

where $a_{m, k}$ and $h_{p, k}$ stand for the $(m, k)$ element of $\mathbf{A}$ and the $(p, k)$ element of $\mathbf{H} . b_{j, k}$ represents the $(j, k)$ element of $\mathbf{B} . n_{m, p, j}$ is the $(m, p, j)$ element of the three - dimensional noise matrix $\mathbf{N}_{x}$. The structure feature of the quadrilinear model in (5) indicates other two rearranged matrix[9] .

$$
\begin{aligned}
& \mathbf{Y}=(\mathbf{H} \circ \mathbf{B}) \mathbf{A}^{T}+\mathbf{N}_{y} . \\
& \mathbf{Z}=(\mathbf{B} \circ \mathbf{A}) \mathbf{H}^{T}+\mathbf{N}_{z} .
\end{aligned}
$$

\section{Compressed PARAFAC Model}

\subsection{PARAFAC model Compression}

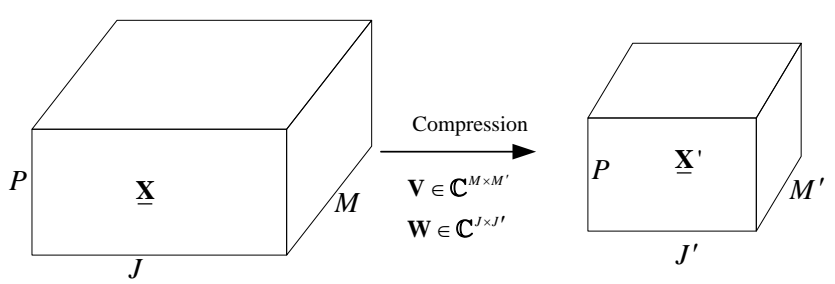

Figure.2 The compression of PARAFAC model

In order to reduce the computational complexity, we firstly compress the three-way data $\overline{\mathbf{X}} \in \mathbb{C}^{P \times J \times M}$ into a much smaller one $\overline{\mathbf{X}} \in \mathbb{C}^{P \times J^{\prime} \times M^{\prime}}$, where $M^{\prime}<M, J^{\prime}<J$. As the row number of $\mathbf{H}$ is small $(P=4)$ and we finally get the two-dimensional angle estimation from $\mathbf{H}$, so in order to improve the algorithm angle estimation performance and avoid sparse reconstruction process, $\mathbf{H}$ would not be compressed. The process is shown in Fig.2. The compressed matrices $\mathbf{W} \in \mathbb{C}^{J \times J^{\prime}}$, and $\mathbf{V} \in \mathbb{C}^{P \times P^{\prime}}$ can be obtained through the Tucker3 decomposition [11] or random special matrices. Then we have

$$
\mathbf{X}^{\prime\left(P \times J^{\prime} M^{\prime}\right)}=\mathbf{X}^{(P \times J M)}(\mathbf{W} \otimes \mathbf{V})+\mathbf{N}_{x}(\mathbf{W} \otimes \mathbf{V}) .
$$

where $\mathbf{X}^{(P \times J M)}=\left[\mathbf{X}_{1}, \mathbf{X}_{2}, \cdots, \mathbf{X}_{M}\right]$. The compressing data can be written as

$$
\mathbf{X}^{\prime}=\left[\mathbf{A}^{\prime} \circ \mathbf{H}\right] \mathbf{B}^{\prime T}+\mathbf{N}_{x}^{\prime} .
$$

where $\mathbf{A}^{\prime}=\mathbf{V}^{H} \mathbf{A}, \mathbf{B}^{\prime}=\mathbf{W}^{H} \mathbf{B}$, and $\mathbf{N}_{x}^{\prime}=\mathbf{N}_{x}(\mathbf{W} \otimes \mathbf{V}) . \mathbf{X}^{\prime}$ can be denoted by the trilinear model or PARAFAC. With respect to (6) and (7), we form the matrices of $\mathbf{Y}^{\prime}$ and $\mathbf{Z}^{\prime}$

$$
\begin{aligned}
& \mathbf{Y}^{\prime}=\left[\mathbf{B}^{\prime} \circ \mathbf{A}^{\prime}\right] \mathbf{H}^{T}+\mathbf{N}_{y}{ }^{\prime} . \\
& \mathbf{Z}^{\prime}=\left[\mathbf{H} \circ \mathbf{B}^{\prime}\right] \mathbf{A}^{\prime T}+\mathbf{N}_{z}{ }^{\prime} .
\end{aligned}
$$

where $\mathbf{N}_{y}{ }^{\prime}$ and $\mathbf{N}_{z}{ }^{\prime}$ are noise.

3.2 PARAFAC decomposition. Trilinear alternating least square (TALS) algorithm is a common data detection method for the trilinear model. The principle of TALS can be adopted for low rank trilinear models. The basic idea of TALS is updating one matrix each time, and the detailed updating procedures are shown as follows [9]:

According to (9), LS fitting is 


$$
\min _{\mathbf{A}^{\prime}, \mathbf{H}, \mathbf{B}^{\prime}}\left\|\tilde{\mathbf{X}}^{\prime}-\left[\mathbf{A}^{\prime} \circ \mathbf{H}\right] \mathbf{B}^{\prime T}\right\|_{F}
$$

where $\|\bullet\|_{F}$ stands for the Forbenius norm. $\odot$ stands for Khatri-Rao product. LS update for $\hat{\mathbf{B}}^{\prime}$ is

$$
\hat{\mathbf{B}}^{\prime T}=\left[\hat{\mathbf{A}}^{\prime} \circ \hat{\mathbf{H}}\right]^{+} \tilde{\mathbf{X}}^{\prime}
$$

where[ ] $]^{+}$is the pseudo-inverse, $\hat{\mathbf{A}}^{\prime}, \hat{\mathbf{H}}$ are previous estimation results of $\mathbf{A}^{\prime}$ and $\mathbf{H}$. Similarly, according to (10) and (11), LS update for $\mathbf{H}$ and $\mathbf{A}^{\prime}$ is

$$
\begin{aligned}
& \hat{\mathbf{H}}^{T}=\left[\hat{\mathbf{B}}^{\prime} \circ \hat{\mathbf{A}}^{\prime}\right]^{+} \tilde{\mathbf{Y}}^{\prime} . \\
& \hat{\mathbf{A}}^{\prime T}=\left[\hat{\mathbf{H}} \circ \hat{\mathbf{B}}^{\prime}\right]^{+} \tilde{\mathbf{Z}}^{\prime} .
\end{aligned}
$$

In order to determine whether the TALS algorithm has converged, we define the sum of squared residuals (SSR) as $S S R=\sum_{i=1}^{P} \sum_{j=1}^{J M}\left|c_{i j}\right|^{2}$, where $c_{i j}$ is the $(i, j)$ element of $\mathbf{C}=\tilde{\mathbf{X}}^{\prime}-\left[\hat{\mathbf{A}}^{\prime} \circ \hat{\mathbf{H}}\right] \hat{\mathbf{B}}^{\prime T}$. According to (13), (14), and (15), we update the matrices $\hat{\mathbf{B}}^{\prime}$, $\hat{\mathbf{H}}$, and $\hat{\mathbf{A}}^{\prime}$, respectively. Until the SSR converges to a certain value, we obtain the final estimation of $\mathbf{B}^{\prime}, \mathbf{H}$, and $\mathbf{A}^{\prime}$.

After the PARAFAC decomposition, the estimated matrices $\hat{\mathbf{H}}$ satisfy [12]

$\hat{\mathbf{H}}=\mathbf{H} \Pi \Delta+\mathbf{E}$.

where $\Pi$ is a permutation matrix, $\Delta$ are diagonal scaling matrices and $\mathbf{E}$ are estimation error. Then we get $\hat{\mathbf{H}}$ normalized and according to (2), we can obtain the automatically paired angle estimation from $\hat{\boldsymbol{h}}_{k}$ as follows:

$$
\begin{aligned}
& \hat{\varphi}_{k}=\sin ^{-1}\left(\hat{\boldsymbol{h}}_{k}(4)\right), k=1,2, \ldots, K . \\
& \hat{\phi}_{k}=\sin ^{-1}\left(\hat{\boldsymbol{h}}_{k}(3) / \cos \left(\hat{\varphi}_{k}\right)\right), \quad k=1,2, \ldots, K .
\end{aligned}
$$

\section{Performance Analysis}

4.1 Complexity analysis. The proposed algorithm needs $O\left\{3 K^{3}+K^{2}\left(J^{\prime}+M^{\prime}+P\right)+6 K^{2}\left(M^{\prime} P+\right.\right.$ $\left.J^{\prime} M^{\prime}+J^{\prime} P\right)$ for each iteration, while the PARAFAC algorithm requires $O\left\{3 K^{3}+K^{2}(J+M+P)+\right.$ $\left.3 K M J P+6 K^{2}(M P+J M+J P)\right\}$ for each iteration [13]. As $M<M^{\prime}, J<J^{\prime}$, The computational complexity of the proposed algorithm is much lower than the conventional PARAFAC algorithm.

4.2 Advantages of the proposed algorithm. The advantages of the proposed algorithm can be summarized as follows.

1) It can achieve automatically paired azimuth and elevation angles estimation.

2) It has lower computational complexity than the conventional PARAFAC algorithm and requires much smaller storage capacity.

3) It has better angle estimation performance than the ESPRIT algorithm and very close performance compared with the conventional PARAFAC algorithm which will be shown in section 5 .

\section{Simulation results}

We conduct Monte Carlo simulations to assess the parameter estimation performance of the 
proposed algorithm. Define root mean square error (RMSE) of the angle and frequency as

$$
R M S E=\frac{1}{K} \sum_{k=1}^{K} \sqrt{\frac{1}{1000} \sum_{l=1}^{1000}\left[\left(\hat{\phi}_{k, l}-\phi_{k}\right)^{2}+\left(\hat{\varphi}_{k, l}-\varphi_{k}\right)^{2}\right]}
$$

where $\phi_{k}$ and $\varphi_{k}$ denote the precise angle of the $k$ th source, and $\hat{\phi}_{k, l}, \hat{\varphi}_{k, l}$ is the estimate of $\phi_{k}, \varphi_{k}$ in the lth Monte Carlo trial, respectively. We assume that there are $K=3$ noncoherent sources with $\left(\phi_{1}, \varphi_{1}\right)=\left(10^{\circ}, 15^{\circ}\right),\left(\phi_{2}, \varphi_{2}\right)=\left(30^{\circ}, 25^{\circ}\right)$, and $\left(\phi_{3}, \varphi_{3}\right)=\left(50^{\circ}, 35^{\circ}\right)$. Note that $M$ is the number of sensors, $J$ is the number of snapshots, and define the compression scale $h=M^{\prime} / M=J^{\prime} / J$.('Proposed' represent for the algorithm we proposed in this paper)

Simulation 1: Fig.3 depict the angle estimation performance of the proposed algorithm with $\mathrm{SNR}=10 \mathrm{~dB}(M=14, J=300$ and $h=0.5)$. The results show that the DOA was clearly observed.

Simulation 2: Fig.4 present the angle estimation performance comparisons with $M=10, J=200$ and $h=0.6$. among the proposed algorithm, the conventional PARAFAC algorithm and the ESPRIT algorithm, we can see that the PARAFAC-based algorithms have better parameter estimation performance than the ESPRIT algorithm. Notably, the proposed algorithm has very close angle estimation performance to the conventional PARAFAC algorithm.

Simulation 3: Fig. 5 shows the angle estimation performance of the proposed algorithm with different values of $J(M=14, h=0.5)$, different values of $M(J=300, h=0.5)$ and different values of $h$ $(M=10, J=300)$, respectively. It is indicated that the angle estimation performance of the proposed algorithm is improved when $M, J$ or $h$ increases. As with $M, J$ or $h$ increasing, we can get more data to estimate more accurate parameter matrices, and the angle estimation performance is enhanced.

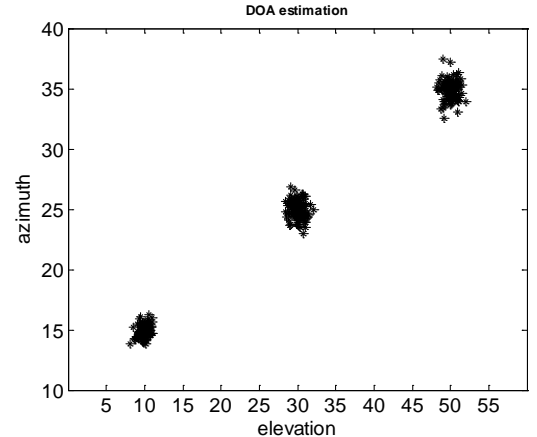

Figure 3. Angle estimation performance

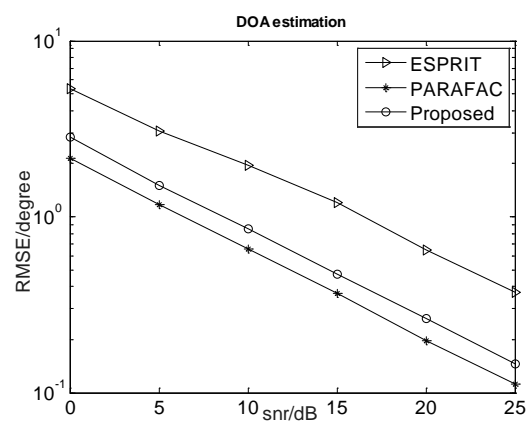

Figure 4. Angle estimation performance comparision
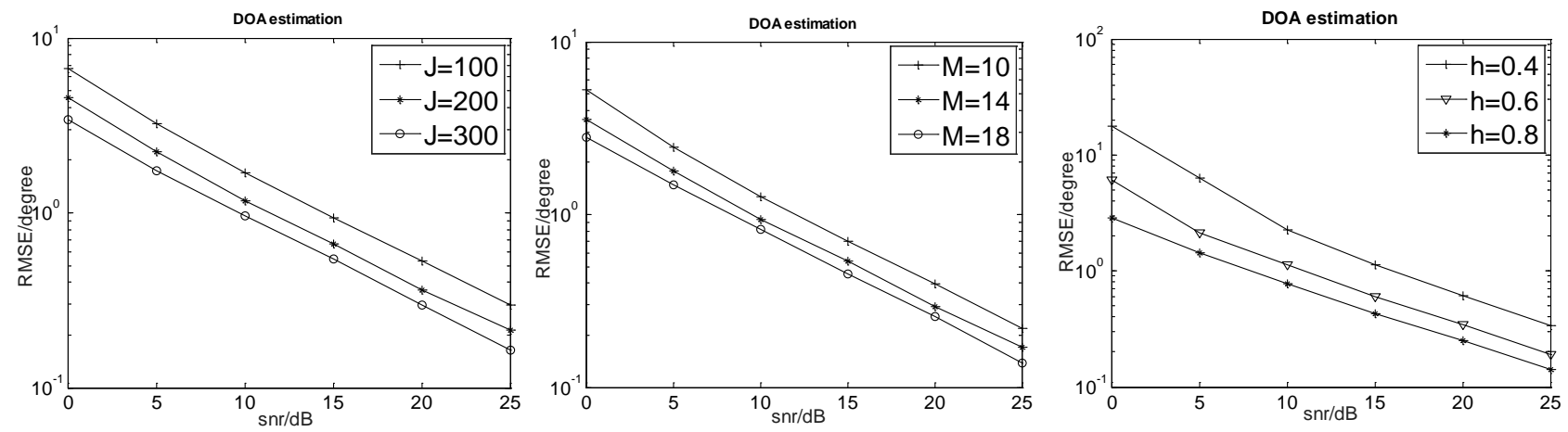

Figure5. Angle estimation performance with different parameter

\section{Summary}

In this work, we proposed a compressed PARAFAC model-based two-dimensional angle estimation algorithm for acoustic vector-sensor arrays. The proposed algorithm compressed the 
PARAFAC model before exploiting the TALS algorithm to estimate the parameter matrices. Owing to compression, the proposed algorithm has smaller storage requirement and lower computational complexity, compared with the conventional PARAFAC algorithm. The angle estimation performance of the proposed algorithm is close to the conventional PARAFAC algorithm, and is better than the ESPRIT algorithm. Numerical simulations illustrate that the proposed work can obtain robust estimation results.

\section{Acknowledgement}

This work is supported by China NSF Grants (61371169, 61601167), the open research fund of National Mobile Communications Research Laboratory, Southeast University (No.2015D030), Jiangsu NSF (BK20161489), the open research fund of State Key Laboratory of Millimeter Waves, Southeast University (No. K201826), and the Fundamental Research Funds for the Central Universities (NO : NE2017103).

\section{References}

[1] Nehorai, A.; Paldi, E. Acoustic vector-sensor array processing. IEEE Trans. Signal Process. 1994,42, 2481-2491.

[2] Hawkes, M.; Nehorai, A. Acoustic vector-sensor beamforming and Capon direction estimation. IEEE Trans. Signal Process. 1998, 46, 2291-2304.

[3] Wong, K.T.; Zoltowski, M.D. Closed-form underwater acoustic direction-finding with arbitrarily spaced vector hydrophones at unknown locations. IEEE J. Ocean. Eng. 1997, 22, 566-575.

[4] Wong, K.T.; Zoltowski, M.D. Root-MUSIC-based azimuth-elevation angle-of-arrival Estimation with uniformly spaced but arbitrarily oriented velocity hydrophones. IEEE Trans. Signal Process.1999, 47, 3250-3260.

[5] Wong, K.T.; Zoltowski, M.D. Self-Initiating MUSIC-based direction finding in underwater acoustic particle velocity-field beamspace. IEEE J. Ocean. Eng. 2000, 25, 262-273.

[6] He, J.; Liu, Z.; Two-dimensional direction finding of acoustic sources by a vector sensor array using the propagator method. Signal Process. 2008, 88, 2492-2499.

[7] Sidiropoulos, N. D., Giannakis, G. B., Bro, R.: 'Blind PARAFAC receivers for DS-CDMA systems', IEEE Trans. Signal Process., 2000, 48, (3), pp. 810-823.

[8] Pham, T.D., Möcks, J.: 'Beyond principal component analysis: a trilinear decomposition model and least squares estimation', Psychometrika, 1992, 57, (2), pp. 203-215

[9] Zhang, X.; Li, J. Trilinear decomposition-based two-dimensional DOA estimation algorithm for arbitrarily spaced acoustic vector-sensor array subjected to unknown locations. Wirel. Pers. Commun. 2012, 67, 859-877.

[10]Sidiropoulos N D, Kyrillidis A. Multi-way compressed sensing for sparse low-rank tensors. IEEE Signal Processing Letters, 2012, 19(11): 757-760.

[11]Vervliet N, Debals O, Sorber L, et al. Breaking the curse of dimensionality using decompositions of incomplete tensors: Tensor-based scientific computing in big data analysis. IEEE, Signal Processing Magazine, 2014, 31(5): 71-79.

[12] Sidiropoulos N D, Giannakis G B, Bro R. Blind PARAFAC receivers for DS-CDMA systems. IEEE Transactions on Signal Processing, 2000, 48(3): 810-823.

[13]Xiaofei Z, Gaopeng F, Jun Y, et al. Angle-frequency estimation using trilinear decomposition of the oversampled output. Wireless personal communications, 2009, 51(2): 365-373. 